Document downloaded from:

http://hdl.handle.net/10251/120547

This paper must be cited as:

Zafar, F.; Cordero Barbero, A.; Quratulain, R.; Torregrosa Sánchez, JR. (2018). Optimal iterative methods for finding multiple roots of nonlinear equations using free parameters. Journal of Mathematical Chemistry. 56(7):1884-1901. https://doi.org/10.1007/s10910-0170813-1

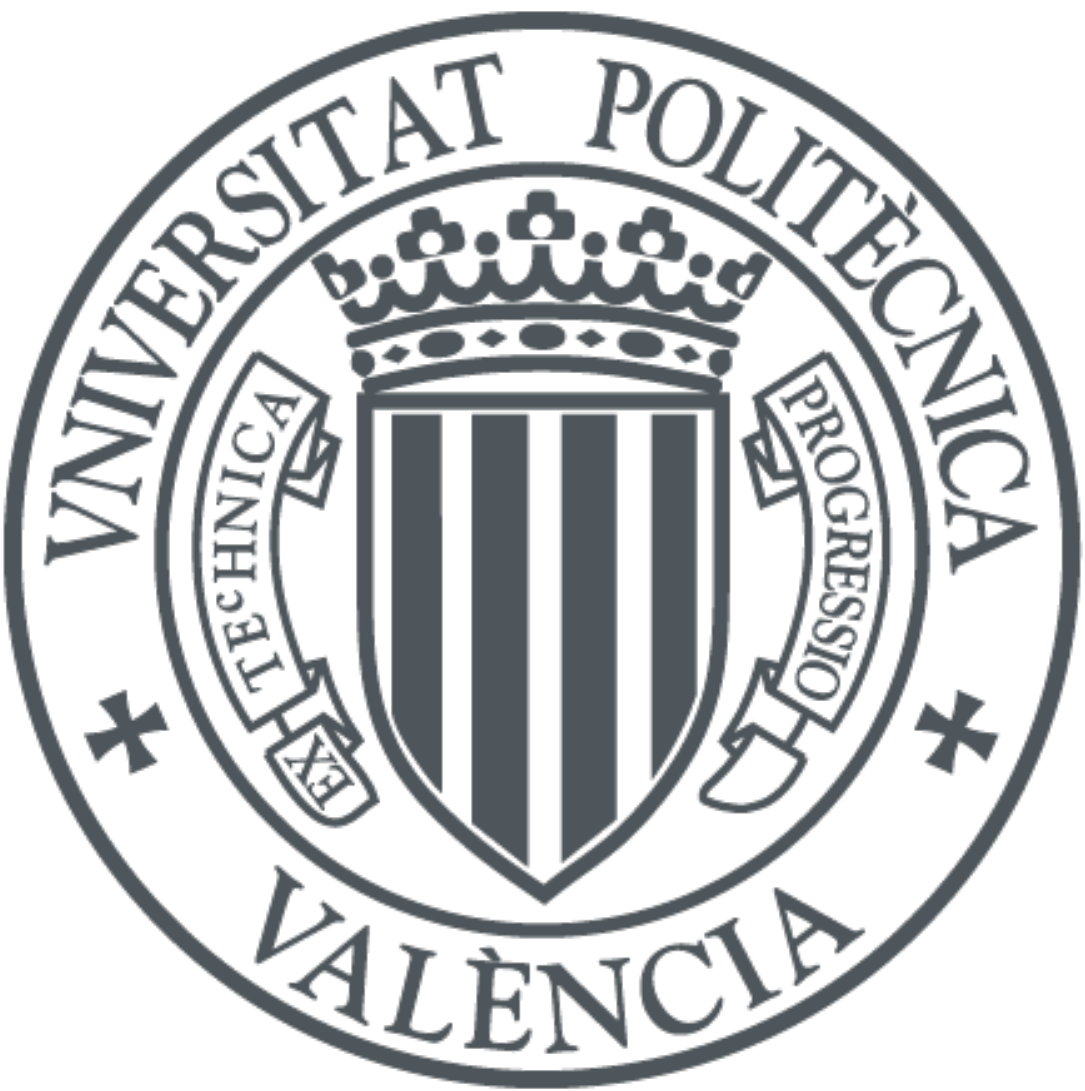

The final publication is available at

http://doi.org/10.1007/s10910-017-0813-1

Copyright Springer-Verlag

Additional Information 


\title{
CMMSE2017-Optimal Iterative Methods for Finding Multiple Roots of Nonlinear Equations using Free Parameters
}

\author{
Fiza Zafar • Alicia Cordero • Quratulain • Juan R. \\ Torregrosa
}

Received: date / Accepted: date

\begin{abstract}
In this paper, we propose a family of optimal eighth order convergent iterative methods for multiple roots with known multiplicity with the introduction of two free parameters and three univariate weight functions. Also numerical experiments have applied to a number of academical test functions and chemical problems for different special schemes from this family that satisfies the conditions given in convergence result.
\end{abstract}

Keywords Iterative methods $\cdot$ nonlinear equations $\cdot$ multiple roots $\cdot$ chemical reactor

\section{Introduction}

Newton's method converges quadratically for every simple root of a nonlinear equation $f(x)=0$. However, if the root has multiplicity $m, m>1$, it is necessary to include a damped parameter, that coincides with the multiplicity, in order to preserve the quadratic convergence.

In past, it was very difficult to construct a higher-order optimal multi-point scheme for multiple zeros of the given function with multiplicity $m \geq 1$. Nowadays, with the digital computer, advanced computer arithmetic, software and symbolic computation, the construction of higher-order optimal multi-point methods has become easy. Many researchers presented optimal fourth-order iterative methods for multiple zeroes like Li et al. [11] in 2009, Sharma and Sharma [16] and Li et al. [12] in 2010, Zhou et al. [21] in 2011, Sharifi et al. [15] in 2012, Soleymani et al. [17], Soleymani and Babajee [18], Liu and Zhou [13] and Zhou et al. [22] in 2013, Thukral [19] in 2014, Behl et al. [1] and Hueso et al. [9] in 2015 and Behl et al. [2] in 2016. In recent years, atmost sixth-order convergence method has been given for finding multiple zeros that can be found in the available literature. There are only three multi-point iterative schemes with sixth-order convergence for multiple zeros. First one was proposed by Thukral [19] and other two were presented by Geum et al. [7,8]. In 2013, Thukral

This research was partially supported by Ministerio de Economía y Competitividad MTM2014-52016-C02-2-P and Generalitat Valenciana PROMETEO/2016/089.

Fiza Zafar

Centre for Advanced Studies in Pure and Applied Mathematics,

Bahauddin Zakariya University, Multan 60800, Pakistan

E-mail: fizazafar@gmail.com

Alicia Cordero

Instituto de Matemática Multidisciplinar,

Universitat Politècnica de València, 46022 Valencia, Spain

E-mail: acordero@mat.upv.es

Quratulain

Centre for Advanced Studies in Pure and Applied Mathematics,

Bahauddin Zakariya University, Multan 60800, Pakistan

E-mail: quratulainrana54@gmail.com

Juan R. Torregrosa

Instituto de Matemática Multidisciplinar,

Universitat Politècnica de València, 46022 Valencia, Spain

E-mail: jrtorre@mat.upv.es 
[20] presented a multi-point iterative method with sixth-order convergence, which is given by:

$$
\begin{aligned}
y_{n} & =x_{n}-m \frac{f\left(x_{n}\right)}{f^{\prime}\left(x_{n}\right)}, \\
z_{n} & =x_{n}-m \frac{f\left(x_{n}\right)}{f^{\prime}\left(x_{n}\right)} \sum_{i=1}^{3}\left(\frac{f\left(y_{n}\right)}{f\left(x_{n}\right)}\right)^{\frac{i}{m}}, \\
x_{n+1} & =z_{n}-m \frac{f\left(x_{n}\right)}{f^{\prime}\left(x_{n}\right)}\left(\frac{f\left(z_{n}\right)}{f\left(x_{n}\right)}\right)^{\frac{1}{m}}\left[\sum_{i=1}^{3}\left(\frac{f\left(y_{n}\right)}{f\left(x_{n}\right)}\right)^{\frac{i}{m}}\right]^{2} .
\end{aligned}
$$

In 2015, Geum et al. [7], have given the following two-point sixth-order iterative scheme:

$$
\begin{aligned}
y_{n} & =x_{n}-m \frac{f\left(x_{n}\right)}{f^{\prime}\left(x_{n}\right)}, m>1, \\
x_{n+1} & =y_{n}-Q\left(p_{n}, s_{n}\right) \frac{f\left(x_{n}\right)}{f^{\prime}\left(x_{n}\right)},
\end{aligned}
$$

where $p_{n}=\sqrt[m]{\frac{f\left(y_{n}\right)}{f\left(x_{n}\right)}}, s_{n}=\sqrt[m-1]{\frac{f^{\prime}\left(y_{n}\right)}{f^{\prime}\left(x_{n}\right)}}$ and $Q: \mathbb{C}^{2} \rightarrow \mathbb{C}$ is holomorphic function in the neighborhood of origin $(0,0)$. In 2016, Geum et al. [8], have again proposed a three-point iterative scheme with sixth-order convergence for multiple zeros. The proposed scheme was based on weight functions, which can be seen in the following expression:

$$
\begin{gathered}
y_{n}=x_{n}-m \frac{f\left(x_{n}\right)}{f^{\prime}\left(x_{n}\right)}, m>1, \\
w_{n}=x_{n}-m G\left(p_{n}\right) \frac{f\left(x_{n}\right)}{f^{\prime}\left(x_{n}\right)}, \\
x_{n+1}=x_{n}-m K\left(p_{n}, t_{n}\right) \frac{f\left(x_{n}\right)}{f^{\prime}\left(x_{n}\right)},
\end{gathered}
$$

where $p_{n}=\sqrt[m]{\frac{f\left(y_{n}\right)}{f\left(x_{n}\right)}}, t_{n}=\sqrt[m]{\frac{f\left(w_{n}\right)}{f\left(x_{n}\right)}}$ and $G: \mathbb{C} \rightarrow \mathbb{C}$ is analytic in a neighborhood of 0 and $K: \mathbb{C}^{2} \rightarrow \mathbb{C}$ is holomorphic in the neighborhood of $(0,0)$. All of the above three schemes (1)-(3) require four function evaluations in order to produce sixth-order convergence. The iterative method (2) has one drawback that it does not work for simple zeros (i.e. for $m=1$ ).

Recently, Behl et al. [3] have developed a family optimal eighth order iterative methods given as:

$$
\begin{gathered}
y_{n}=x_{n}-m \frac{f\left(x_{n}\right)}{f^{\prime}\left(x_{n}\right)}, m>1, \\
z_{n}=y_{n}-u_{n} Q\left(h_{n}\right) \frac{f\left(x_{n}\right)}{f^{\prime}\left(x_{n}\right)}, \\
z_{n+1}=x_{n}-u_{n} t_{n} G\left(h_{n}, t_{n}\right) \frac{f\left(x_{n}\right)}{f^{\prime}\left(x_{n}\right)},
\end{gathered}
$$

where $u_{n}=\sqrt[m]{\frac{f\left(y_{n}\right)}{f\left(x_{n}\right)}}, h_{n}=\frac{u_{n}}{a_{1}+a_{2} u_{n}}, t_{n}=\sqrt[m]{\frac{f\left(z_{n}\right)}{f\left(y_{n}\right)}}$ and $Q: \mathbb{C} \rightarrow \mathbb{C}$ is analytic in a neighborhood of 0 and $G: \mathbb{C}^{2} \rightarrow \mathbb{C}$ is holomorphic in the neighborhood of $(0,0)$.

Motivated by the research going on in this direction and with a need to give more optimal higher order methods, we propose an optimal eighth-order convergent iterative method for multiple root of a nonlinear equation. The main reason of this proposed method is to present a new higher-order optimal scheme for finding simple as well as multiple zeros of nonlinear equations.

The rest of the paper is organized as follows: In Section 2, we propose a new family of optimal eighthorder iterative methods to find multiple roots of nonlinear equation and discuss its convergence analysis. Some special cases are given in the Section 3. In Section 4, numerical performance and comparison of the proposed schemes with the existing ones are given. Academic test functions and nonlinear equations that appear in different chemical problems such as Van der Waals equation, fractional conversion in a chemical reactor and the isothermal continuous stirred tank reactor are used in this section. Concluding remarks are given in Section 5. 


\section{Construction of the scheme}

This section is devoted to the construction and convergence analysis of this proposed scheme with the main theorem. So, we propose a new eighth-order scheme for a known multiplicity $m \geq 1$ of the desired multiple zero as follows

$$
\begin{aligned}
y_{n} & =x_{n}-m \frac{f\left(x_{n}\right)}{f^{\prime}\left(x_{n}\right)}, \\
z_{n} & =y_{n}-m u_{n} H\left(u_{n}\right) \frac{f\left(x_{n}\right)}{f^{\prime}\left(x_{n}\right)}, \\
x_{n+1} & =z_{n}-u_{n} v_{n}\left(A_{2}+A_{3} u_{n}\right) P\left(v_{n}\right) G\left(w_{n}\right) \frac{f\left(x_{n}\right)}{f^{\prime}\left(x_{n}\right)},
\end{aligned}
$$

where $A_{2}, A_{3} \in R$ are free parameters and the weight functions $H: \mathbb{C} \rightarrow \mathbb{C}, P: \mathbb{C} \rightarrow \mathbb{C}, G: \mathbb{C} \rightarrow \mathbb{C}$ are analytic function in the neighborhood of 0 with

$$
u_{n}=\left(\frac{f\left(y_{n}\right)}{f\left(x_{n}\right)}\right)^{\frac{1}{m}}, \quad v_{n}=\left(\frac{f\left(z_{n}\right)}{f\left(y_{n}\right)}\right)^{\frac{1}{m}}, \quad w_{n}=\left(\frac{f\left(z_{n}\right)}{f\left(x_{n}\right)}\right)^{\frac{1}{m}},
$$

are the weight functions. It is worthy to note that we will obtain well known King's family of fourth-order iterative methods for $m=1$ with the help of first two substeps. In the next result, we demonstrate that the order of convergence of the proposed scheme will reach at optimal eight without using additional functional evaluations.

Theorem 1 Let $x=\alpha$ be a multiple zero with a multiplicity $m \geq 1$ of the involved function $f$. In addition, we assume that $f: \mathbb{C} \rightarrow \mathbb{C}$ is an analytic function in the region enclosing a multiple zero $\alpha$. The proposed scheme defined by (5) has an optimal eighth-order convergence, when it satisfies the following conditions:

$$
\begin{aligned}
& A_{2}=1, A_{3}=2 A_{2}, \\
& H_{0}=H(0)=1, H_{1}=H^{\prime}(0)=2, H_{2}=H^{\prime \prime}(0)=-2, H_{3}=H^{\prime \prime \prime}(0)=36, \\
& P_{0}=P(0)=P^{\prime}(0), G_{0}=G(0)=\frac{m}{P_{0} A_{2}}, G_{1}=G^{\prime}(0)=\frac{2 m}{P_{0} A_{2}}
\end{aligned}
$$

and the error equation is given as:

$$
\begin{aligned}
e_{n+1}= & \left.\frac{1}{48 m^{7} P_{0}} c_{1} c_{2}^{2}(11+m)-2 m c_{2}\right)\left(\left(14\left(59+12 m+m^{2}\right) P_{0}-3(11+m)^{2} P_{2}\right) c_{1}^{4}\right. \\
& \left.-12 m\left(4(7+m) P_{0}-(11+m)\right) c_{1}^{2} c_{2}+12 m^{2}\left(2 P_{0}-P_{2}\right) c_{2}^{2}+24 m^{2} P_{0} c_{1} c_{3}\right) e_{n}^{8} \\
& +O\left(e_{n}^{9}\right)
\end{aligned}
$$

where $e_{n}=x_{n}-\alpha$ and $c_{k}=\frac{1}{k !} \frac{f^{(k)}(\alpha)}{f^{\prime}(\alpha)}, i=1,2, \ldots$

Proof Let $x=\alpha$ be a multiple zero of $f(x)$. Expanding $f\left(x_{n}\right)$ and $f^{\prime}\left(x_{n}\right)$ about $x=\alpha$ by the Taylor's series expansion, we obtain

$$
f\left(x_{n}\right)=\frac{f^{(m)}(\alpha)}{m !} e_{n}^{m}\left(1+c_{1} e_{n}+c_{2} e_{n}^{2}+c_{3} e_{n}^{3}+c_{4} e_{n}^{4}+c_{5} e_{n}^{5}+c_{6} e_{n}^{6}+c_{7} e_{n}^{7}+c_{8} e_{n}^{8}+O\left(e_{n}^{9}\right)\right),
$$

and

$$
\begin{aligned}
f^{\prime}\left(x_{n}\right)= & \frac{f^{(m)}(\alpha)}{m !} e_{n}^{m-1}\left(m+c_{1}(m+1) e_{n}+c_{2}(m+2) e_{n}^{2}+c_{3}(m+3) e_{n}^{3}+c_{4}(m+4) e_{n}^{4}\right. \\
& \left.+c_{5}(m+5) e_{n}^{5}+c_{6}(m+6) e_{n}^{6}+c_{7}(m+7) e_{n}^{7}+c_{8}(m+8) e_{n}^{8}+O\left(e_{n}^{9}\right)\right)
\end{aligned}
$$

respectively. By using expressions (7) and (8) in the first substep of (5), we obtain

$$
y_{n}-\alpha=\frac{c_{1} e_{n}^{2}}{m}+\frac{\left(2 c_{2} m-c_{1}^{2}(m+1)\right) e_{n}^{3}}{m^{2}}+\sum_{k=0}^{4} B_{k} e_{n}^{k+4}+O\left(e_{n}^{9}\right),
$$


where $B_{k}=B_{k}\left(m, c_{1}, c_{2}, \ldots, c_{8}\right)$ are expressed in terms of $m, c_{2}, c_{3}, \ldots, c_{8}$, where the two coefficients $B_{0}$ and $B_{1}$ can be explicitly written as $B_{0}=\frac{1}{m^{3}}\left\{3 c_{3} m^{2}+c_{1}^{3}(m+1)^{2}-c_{1} c_{2} m(3 m+4)\right\}$ and $B_{1}=-\frac{1}{m^{4}}\left\{c_{1}^{4}(m+1)^{3}-\right.$ $\left.2 c_{2} c_{1}^{2} m\left(2 m^{2}+5 m+3\right)+2 c_{3} c_{1} m^{2}(2 m+3)+2 m^{2}\left(c_{2}^{2}(m+2)-2 c_{4} m\right)\right\}$, etc. With the help of Taylor's series expansion, we obtain

$$
f\left(y_{n}\right)=f^{(m)}(\alpha) e_{n}^{2 m}\left[\frac{\left(\frac{c_{1}}{m}\right)^{m}}{m !}+\frac{\left(2 m c_{2}-(m+1) c_{1}^{2}\right)\left(\frac{c_{1}}{m}\right)^{m} e_{n}}{c_{1} m !}+\sum_{k=0}^{6} \bar{B}_{k} e_{n}^{k+2}+O\left(e_{n}^{9}\right)\right] .
$$

By using the expressions (7) and (10), we get

$$
u_{n}=\frac{c_{1} e_{n}}{m}+\frac{\left(2 m c_{2}-(m+2) c_{1}^{2}\right) e_{n}^{2}}{m^{2}}+\tau_{1} e_{n}^{3}+\tau_{2} e_{n}^{4}+\tau_{3} e_{n}^{5}+O\left(e_{n}^{6}\right),
$$

where,

$$
\begin{gathered}
\tau_{1}=\frac{1}{2 m^{3}}\left[c_{1}^{3}\left(2 m^{2}+7 m+7\right)+6 c_{3} m^{2}-2 c_{1} c_{2} m(3 m+7)\right] \\
\tau_{2}=-\frac{1}{6 m^{4}}\left[c_{1}^{4}\left(6 m^{3}+29 m^{2}+51 m+34\right)-6 c_{2} c_{1}^{2} m\left(4 m^{2}+16 m+17\right)+12 c_{1} c_{3} m^{2}(2 m+5)\right. \\
\left.+12 m^{2}\left(c_{2}^{2}(m+3)-2 c_{4} m\right)\right] \\
\tau_{3}=\frac{1}{24 m^{5}}\left[-24 m^{3}\left(c_{2} c_{3}(5 m+17)-5 c_{5} m\right)+12 c_{3} c_{1}^{2} m^{2}\left(10 m^{2}+43 m+49\right)\right. \\
+12 c_{1} m^{2}\left\{c_{2}^{2}\left(10 m^{2}+47 m+53\right)-2 c_{4} m(5 m+13)\right\}-4 c_{2} c_{1}^{3} m\left(30 m^{3}+163 m^{2}+306 m+209\right) \\
\left.+c_{1}^{5}\left(24 m^{4}+146 m^{3}+355 m^{2}+418 m+209\right)\right]
\end{gathered}
$$

Expanding Taylor series of $H(u)$ about 0, we have:

$$
H(u) \approx H_{0}+H_{1} u+\frac{H_{2}}{2 !} u^{2}+\frac{H_{3}}{3 !} u^{3},
$$

where $H_{j}=H^{(j)}(0)$ for $0 \leq j \geq 3$. Inserting expressions (9)-(12) in the second substep of scheme (5), we have

$$
\begin{aligned}
z_{n}= & \alpha+\frac{\left(-1+H_{0}\right) c_{1} e_{n}^{2}}{m}-\frac{\left.\left(1+H_{1}+m-H_{0}(3+m) c_{1}^{2}\right)+2\left(-1+H_{0}\right) m c_{2}\right) e_{n}^{3}}{m^{2}} \\
& +\frac{1}{2 m^{3}}\left[\left(2+10 H_{1}-H_{2}+4 m+4 H_{1} m+2 m^{2}-H_{0}\left(13+11 m+2 m^{2}\right)\right) c_{1}^{3}\right. \\
& +2 m\left(-4-4 H_{1}-3 m+H_{0}(11+3 m) c_{1} c_{2}-6\left(-1+H_{0}\right) m^{2} c_{3}\right) e_{n}^{4} \\
& \left.+z_{5} e_{n}^{5}+z_{6} e_{n}^{6}+z_{7} e_{n}^{7}+O\left(e_{n}^{8}\right)\right] .
\end{aligned}
$$

By selecting $H_{0}=1$ and $H_{1}=2$, we obtain

$$
z_{n}=\alpha+\frac{c_{1}^{3}\left(9-H_{2}+m\right)-2 m c_{1} c_{2}}{2 m^{3}} e_{n}^{4}+z_{5} e_{n}^{5}+z_{6} e_{n}^{6}+z_{7} e_{n}^{7}+O\left(e_{n}^{8}\right),
$$

where

$$
\begin{aligned}
z_{5}=-\frac{1}{6 m^{4}}\{ & \left.c_{1}^{4}\left(125+H_{3}+84 m+7 m^{2}-3 H_{2}(7+3 m)+6 m\left(-3 H_{2}+4(7+m)\right) c_{1}^{2} c_{2}+12 c_{2}^{2} m^{2}+12 c_{2} c_{1} m\right)\right\} \\
z_{6}= & \frac{1}{24 m^{5}}\left\{1507+1850 m+677 m^{2}+46 m^{3}+4 H_{3}(9+4 m)-6 H_{2}\left(59+53 m+12 m^{2}\right)\right) c_{1}^{5} \\
& -4 m\left(925+8 H_{3}+594 m+53 m^{2}-3 H_{2}(53+21 m) c_{1}^{3} c_{2}+12 m^{2}\left(83-9 H_{2}+13 m\right) c_{1}^{2} c_{3}\right. \\
& \left.\left.-168 m^{3} c_{2} c_{3}+12 m^{2} c_{1}\left(115-12 H_{2}+17 m\right) c_{2}^{2}-6 m c_{4}\right)\right\},
\end{aligned}
$$

and

$$
\begin{aligned}
z_{7}= & -\left\{12 c_{1}^{2} c_{3} m^{2}(36 \beta+13 m+11)+\left(37-168 c_{2} c_{3} m^{3}+4 c_{1}^{3} c_{2} m\left(96 \beta^{2}+252 \beta+53 m^{2}\right.\right.\right. \\
& \left.+18(14 \beta+5) m)+12 c_{1} m^{2}\left(c_{2}^{2}(48 \beta+17 m+19)-6 c_{4} m\right)\right\} .
\end{aligned}
$$


Now, again by using the Taylor's series expansion for (13), we have

$$
\begin{aligned}
f\left(z_{n}\right)= & f^{(m)}(\alpha) e_{n}^{4 m}\left[\frac{2^{-m}\left(\frac{c_{1}^{3}\left(9-H_{2}+m\right)-2 m c_{1} c_{2}}{m^{3}}\right)^{m}}{m !}-\frac{\left(2^{-m}\left(\frac{c_{1}^{3}\left(9-H_{2}+m\right)-2 m c_{1} c_{2}}{m^{3}}\right)^{m-1} \rho_{0}\right)}{3\left(m^{3} m !\right)} e_{n}\right. \\
& \left.+\sum_{j=0}^{7} \bar{H}_{j} e_{n}^{j+1}+O\left(e_{n}^{9}\right)\right],
\end{aligned}
$$

where $\left.\rho_{0}=c_{1}^{4}\left(125+H_{3}+84 m+7 m^{2}-3 H_{2}(7+3 m)\right) c_{1}^{4}-6 m\left(-3 H_{2}+4(7+m)\right) c_{1}^{2} c_{2}+12 m^{2} c_{2}^{2}+12 c_{3} c_{1} m^{2}\right)$. With the help of expressions (7) and (14), we have

$$
v_{n}=\frac{c_{1}^{2}\left(9-H_{2}+m\right)-2 m c_{2}}{2 m^{2}} e_{n}^{2}+\rho_{1} e_{n}^{3}+\rho_{2} e_{n}^{4}+\rho_{3} e_{n}^{5}+O\left(e_{n}^{6}\right),
$$

where,

$$
\begin{aligned}
\rho_{1}=- & \frac{1}{6 m^{3}}\left\{c_{1}^{3}\left(98+H_{3}+4 m^{2}+54 m-6 H_{2}(3+m)-12 m\left(9-H_{2}+m\right) c_{1} c_{2}+12 m^{2} c_{3}\right\},\right. \\
\rho_{2}= & \left.\frac{1}{24 m^{4}} 899+1002 m+313 m^{2}+18 m^{3}+4 H_{3}(8+3 m)-6 H_{2}\left(43+33 m+6 m^{2}\right)\right) c_{1}^{4} \\
& -12 m\left(167+2 H_{3}+87 m+6 m^{2}-H_{2}(33+10 m) c_{1}^{2} c_{2}\right. \\
& +24 m^{2}\left(26-3 H_{2}+3 m\right) c_{1} c_{3}+12 m^{2}\left(c_{2}^{2}\left(35-4 H_{2}+3 m\right)-6 m c_{4}\right),
\end{aligned}
$$

etc.

Also,

$$
w_{n}=\left(\frac{f\left(z_{n}\right)}{f\left(x_{n}\right)}\right)^{\frac{1}{m}}=\frac{1}{2 m^{3}}\left\{c_{1}^{3}\left(m-H_{2}+9\right)-2 m c_{1} c_{2}\right\} e_{n}^{3}+\sum_{i=1}^{4} \sigma_{i} e_{n}^{i+3}+O\left(e_{n}^{8}\right)
$$

where $\sigma_{i}=\sigma_{i}\left(m, c_{1}, c_{2}, \ldots, c_{8}\right), 1 \leq i \leq 4$, and the first two coefficients are

$$
\begin{aligned}
\sigma_{1}= & \frac{-1}{6 m^{4}}\left[c_{1}^{4}\left\{7 m^{2}+87 m+H_{3}+152-3 H_{2}(3 m+8)\right\}-6 m c_{1}^{2} c_{2}\left(4 m-3 H_{2}+29\right)+12 m^{2} c_{2}^{2}\right. \\
& \left.+12 m^{2} c_{1} c_{3}\right], \\
\sigma_{2}= & \frac{1}{24 m^{5}}\left[c_{1}^{5}\left\{46 m^{3}+711 m^{2}+2246 m+2061+8 H_{3}(2 m+5)-12 H_{2}\left(6 m^{2}+30 m+37\right)\right\}\right. \\
& -4 m c_{1}^{3}\left\{53 m^{2}+624 m+8 H_{3}+1123-9 H_{2}(7 m+20)\right\}+12 m^{2} c_{1}^{2} c_{3}\left(13 m-9 H_{2}+87\right) \\
& \left.-168 m^{3} c_{2} c_{3}+12 m^{2} c_{1}\left\{c_{2}^{2}\left(17 m-12 H_{2}+121\right)-6 m c_{4}\right\}\right]
\end{aligned}
$$

Expanding weight functions $P(v)$ and $G(w)$ in the neighborhood of origin by Taylor's series expansion as follows:

$$
\begin{aligned}
P(v) & \approx P_{0}+P_{1} v+P_{2} \frac{v^{2}}{2}+P_{3} \frac{v^{3}}{6} \\
G(w) & \approx G+G_{1} w+G_{2} \frac{w^{2}}{2}+G_{3} \frac{w^{3}}{6}
\end{aligned}
$$

By using expressions (7)-(18) in the proposed scheme (5), we have

$$
e_{n+1}=\frac{1}{2 m^{4}}\left(m-P_{0} G_{0} A_{2}\right)\left(\left(9-H_{2}+m\right) c_{1}^{2}-2 m c_{2}\right) c_{1} e_{n}^{4}+O\left(e_{n}^{5}\right) .
$$

For obtaining at least sixth-order convergence, we have to choose $G_{0}=\frac{m}{P_{0} A_{2}}, A_{3}=2 A_{2}, P_{1}=P_{0}$ and get

$$
e_{n+1}=-\frac{1}{4 m^{5}}\left(2+H_{2}\right)\left(\left(9-H_{2}+m\right) c_{1}^{2}-2 m c_{2}\right) c_{1}^{3} e_{n}^{6}+O\left(e_{n}^{7}\right) .
$$


Further, in order to obtain eighth order of convergence we choose the following values of parameters:

$$
H_{2}=-2, H_{3}=36, G_{1}=\frac{2 m}{P_{0} A_{2}}
$$

which leads us to the following error equation:

$$
\begin{aligned}
e_{n+1}= & \frac{1}{48 m^{7} P_{0}}\left[\left(c_{1} c_{1}^{2}(11+m)-2 m c_{2}\right)\left(14\left(59+12 m+m^{2}\right) P_{0}-3(11+m)^{2} P_{2}\right) c_{1}^{4}\right. \\
& \left.\left.-12 m\left(4(7+m) P_{0}-(11+m)\right) c_{1}^{2} c_{2}+12 m^{2}\left(2 P_{0}-P_{2}\right) c_{2}^{2}+24 m^{2} P_{0} c_{1} c_{3}\right) e_{n}^{8}+O\left(e_{n}^{9}\right)\right] .
\end{aligned}
$$

The above asymptotic error constant (21) reveals that the proposed scheme (5) reaches to optimal eighthorder convergence by using only four functional evaluations (viz. $f\left(x_{n}\right), f^{\prime}\left(x_{n}\right), f\left(y_{n}\right)$ and $f\left(z_{n}\right)$ ) per iteration. This completes the proof.

\section{Some special cases of weight function}

In this section, we will discuss some special cases of our proposed scheme (5) by assigning different kind of weight functions. In this regard, please see following cases, where we have mentioned some different kind of choices for the proposed scheme:

Case 1 Let us describe the following polynomial weight function directly from the proposed Theorem 1:

$$
H(u)=6 u_{n}^{3}-u_{n}^{2}+2 u_{n}+1, P\left(v_{n}\right)=v_{n}+1, G\left(w_{n}\right)=\frac{2 m w_{n}}{A_{2} P_{0}}+\frac{m}{A_{2} P_{0}}
$$

Thus, the corresponding optimal eighth-order iterative scheme is given by

$$
\begin{aligned}
y_{n} & =x_{n}-m \frac{f\left(x_{n}\right)}{f^{\prime}\left(x_{n}\right)}, \\
z_{n} & =y_{n}-m u_{n}\left(6 u_{n}^{3}-u_{n}^{2}+2 u_{n}+1\right) \frac{f\left(x_{n}\right)}{f^{\prime}\left(x_{n}\right)}, \\
x_{n+1} & =z_{n}-m u_{n} v_{n}\left(1+2 u_{n}\right)\left(1+v_{n}\right)\left(\frac{2 w_{n}+1}{A_{2} P_{0}}\right) \frac{f\left(x_{n}\right)}{f^{\prime}\left(x_{n}\right)} .
\end{aligned}
$$

Case 2 Now, we suggest mixture of rational and polynomial weight function satisfying the conditions as follows

$$
H\left(u_{n}\right)=\frac{1-5 u_{n}^{2}+8 u_{n}^{3}}{-2 u_{n}+1}, P\left(v_{n}\right)=v_{n}+1, G(w)=\frac{3 m w_{n}+m}{A_{2} P_{0}\left(1+w_{n}\right)} .
$$

Thus, the corresponding optimal eighth-order iterative scheme is given by

$$
\begin{aligned}
y_{n} & =x_{n}-m \frac{f\left(x_{n}\right)}{f^{\prime}\left(x_{n}\right)}, \\
z_{n} & =y_{n}-m u_{n}\left(\frac{1-5 u_{n}^{2}+8 u_{n}^{3}}{-2 u_{n}+1}\right) \frac{f\left(x_{n}\right)}{f^{\prime}\left(x_{n}\right)}, \\
x_{n+1} & =z_{n}-m u_{n} v_{n}\left(1+2 u_{n}\right)\left(v_{n}+1\right)\left(\frac{3 w_{n}+1}{A_{2} P_{0}\left(1+w_{n}\right)}\right) \frac{f\left(x_{n}\right)}{f^{\prime}\left(x_{n}\right)} .
\end{aligned}
$$

Case 3 Moreover, a mixture of polynomial and rational function is given as:

$$
H\left(u_{n}\right)=\frac{1-5 u_{n}^{2}+8 u_{n}^{3}}{-2 u_{n}+1}, P\left(v_{n}\right)=v_{n}+1, G\left(w_{n}\right)=\frac{2 m w_{n}+m}{A_{2} P_{0}} .
$$

Thus, the corresponding optimal eighth-order iterative scheme is given by

$$
\begin{aligned}
y_{n} & =x_{n}-m \frac{f\left(x_{n}\right)}{f^{\prime}\left(x_{n}\right)} \\
z_{n} & =y_{n}-m u_{n}\left(\frac{1-5 u_{n}^{2}+8 u_{n}^{3}}{-2 u_{n}+1}\right) \frac{f\left(x_{n}\right)}{f^{\prime}\left(x_{n}\right)}, \\
x_{n+1} & =z_{n}-m u_{n} v_{n}\left(1+2 u_{n}\right)\left(v_{n}+1\right)\left(\frac{2 w_{n}+1}{A_{2} P_{0}}\right) \frac{f\left(x_{n}\right)}{f^{\prime}\left(x_{n}\right)} .
\end{aligned}
$$


Case 4 Mixture of polynomial and exponential function as

$$
H\left(u_{n}\right)=6 u_{n}^{3}-u_{n}^{2}+2 u_{n}+1, P\left(v_{n}\right)=e^{v_{n}}, G\left(w_{n}\right)=\frac{m e^{2 w_{n}}}{A_{2} P_{0}}
$$

Thus, the corresponding optimal eighth-order iterative scheme is given by

$$
\begin{aligned}
y_{n} & =x_{n}-m \frac{f\left(x_{n}\right)}{f^{\prime}\left(x_{n}\right)}, \\
z_{n} & =y_{n}-m u_{n}\left(6 u_{n}^{3}-u_{n}^{2}+2 u_{n}+1\right) \frac{f\left(x_{n}\right)}{f^{\prime}\left(x_{n}\right)}, \\
x_{n+1} & =z_{n}-m u_{n} v_{n} e^{v_{n}}\left(1+2 u_{n}\right)\left(\frac{e^{2 w_{n}}}{A_{2} P_{0}}\right) \frac{f\left(x_{n}\right)}{f^{\prime}\left(x_{n}\right)} .
\end{aligned}
$$

Case 5 Mixture of polynomial, exponential and rational function is given as:

$$
H\left(u_{n}\right)=6 u_{n}^{3}-u_{n}^{2}+2 u_{n}+1, P\left(v_{n}\right)=e^{v_{n}}, G\left(w_{n}\right)=\frac{2 m w_{n}+m}{A_{2} P_{0}}
$$

Thus, the corresponding optimal eighth-order iterative scheme is given by

$$
\begin{aligned}
y_{n} & =x_{n}-m \frac{f\left(x_{n}\right)}{f^{\prime}\left(x_{n}\right)}, \\
z_{n} & =y_{n}-m u_{n}\left(6 u_{n}^{3}-u_{n}^{2}+2 u_{n}+1\right) \frac{f\left(x_{n}\right)}{f^{\prime}\left(x_{n}\right)}, \\
x_{n+1} & =z_{n}-m u_{n} v_{n} e^{v_{n}}\left(1+2 u_{n}\right)\left(\frac{2 w_{n}+1}{A_{2} P_{0}}\right) \frac{f\left(x_{n}\right)}{f^{\prime}\left(x_{n}\right)} .
\end{aligned}
$$

Case 6 Mixture of polynomials and rational function as

$$
H\left(u_{n}\right)=6 u_{n}^{3}-u_{n}^{2}+2 u_{n}+1, P\left(v_{n}\right)=v_{n}+1, G\left(w_{n}\right)=\frac{3 m w_{n}+m}{A_{2} P_{0}\left(1+w_{n}\right)}
$$

Thus, the corresponding optimal eighth-order iterative scheme is given by

$$
\begin{aligned}
y_{n} & =x_{n}-m \frac{f\left(x_{n}\right)}{f^{\prime}\left(x_{n}\right)}, \\
z_{n} & =y_{n}-m u_{n}\left(6 u_{n}^{3}-u_{n}^{2}+2 u_{n}+1\right) \frac{f\left(x_{n}\right)}{f^{\prime}\left(x_{n}\right)}, \\
x_{n+1} & =z_{n}-m u_{n} v_{n}\left(1+2 . u_{n}\right)\left(v_{n}+1\right)\left(\frac{3 w_{n}+1}{A_{2} P_{0}\left(1+w_{n}\right)}\right) \frac{f\left(x_{n}\right)}{f^{\prime}\left(x_{n}\right)} .
\end{aligned}
$$

\section{Numerical experiments}

This section is devoted to demonstrate the efficiency, effectiveness and convergence behavior of the presented schemes. In this regard, we consider some of the special cases of the proposed scheme namely expression (23)(29) denoted by M1, M2, M3 and M4 respectively, with $A_{2}=P_{0}=1$. In addition, we choose a total number of four test problems for comparison given in the Examples 1-4. Now, we want to compare our methods with other existing methods of same domain on the basis of error per iteration and computational order of convergence COC. We compare the proposed methods with the family of two-point sixth-order methods, which were presented by Geum et al. in [8], out of them we consider (2) and (3) denoted by GM1 and GM2 respectively, for $Q\left(p_{n}, s_{n}\right)=$ $m\left(1+2(m-1)\left(p_{n}-t_{n}\right)-4 p_{n} t_{n}+t_{n}^{2}\right), G\left(p_{n}\right)=m\left(1+p_{n}+2 p_{n}^{2}\right)$, and $K\left(p_{n}, t_{n}\right)=m\left(1+p_{n}+2 p_{n}^{2}+\left(1+2 p_{n}\right) t_{n}\right)$ and finally we choose a special case of the optimal eighth order method given by Behl et al. [3] for $a_{1}=a_{2}=1$, $Q\left(h_{n}\right)=m\left(1+2 h_{n}+3 h_{n}^{2}\right)$ and $G\left(h_{n}, t_{n}\right)=m \frac{1+2 t_{n}+3 h_{n}^{2}+h_{n}\left(2+6 t_{n}+h_{n}\right)}{1+t_{n}}$ in $(4)$ denoted by OM . In Table 1 , we choose first four test problems of weight functions for comparison: we display the number of iteration indexes $n$, 
Table 1: Results for test functions $f_{1}(x)-f_{4}(x)$ for selected new method

\begin{tabular}{|c|c|c|c|c|c|c|c|}
\hline Methods & $f_{i}(x)$ & $n$ & $x_{n}$ & $\left|f\left(x_{n}\right)\right|$ & $\left|x_{n}-a\right|$ & $\left|e_{n} / e_{n-1}^{8}\right|$ & $\rho_{n}$ \\
\hline \multirow[t]{4}{*}{$M 1$} & $f_{1}$ & 0 & 1.8 & & & & \\
\hline & & 1 & 1.750388172 & $4.578821428(-9)$ & $3.881723198(-4)$ & & \\
\hline & & 2 & 1.750000000 & $7.990332601(-35)$ & $5.160856712(-17)$ & $1.057651891(7)$ & 5.551003268 \\
\hline & & 3 & 1.750000000 & $1.054997370(-240)$ & $5.930141567(-120)$ & $1.001210272(11)$ & 7.992771335 \\
\hline \multirow[t]{4}{*}{$M 2$} & $f_{2}$ & 0 & 0.76 & & & & \\
\hline & & 1 & 0.757396246 & $6.119297566(-9)$ & $7.672103880(-11)$ & & \\
\hline & & 2 & 0.757396246 & $4.708676719(-69)$ & $5.903530034(-71)$ & $3.631744725(10)$ & 7.963102504 \\
\hline & & 3 & 0.757396246 & $5.787317358(-550)$ & $7.255881828(-552)$ & $4.918084433(10)$ & 7.999999999 \\
\hline \multirow{4}{*}{ M3 } & $f_{3}$ & 0 & -3.0 & & & & \\
\hline & & 1 & -2.840827595 & $1.765950473(-4)$ & $9.172404158(-3)$ & & \\
\hline & & 2 & -2.850019022 & $7.599195264(-10)$ & $1.902277880(-5)$ & $7.279720405(-4)$ & 2.212077357 \\
\hline & & 3 & -2.850000000 & $7.150328989(-83)$ & $5.835168006(-42)$ & $1.058646197(-12)$ & 13.6085671491 \\
\hline \multirow[t]{4}{*}{$M 4$} & $f_{4}$ & 0 & 1.0 & & & & \\
\hline & & 1 & 0.739085163 & $1.263052195(-22)$ & $2.997912648(-8)$ & & \\
\hline & & 2 & 0.739085133 & $4.433422964(-187)$ & $4.556082715(-63)$ & $1.395817915(-3)$ & 7.873921103 \\
\hline & & 3 & 0.739085133 & $1.021603664(-1502)$ & $1.296500510(-501)$ & $6.982964029(-3)$ & 7.999999998 \\
\hline
\end{tabular}

the error at each iterations $\left|x_{n}-\alpha\right|$, the functional value at $x_{n},\left|f\left(x_{n}\right)\right|$, the asymptotic error constant $\left|e_{n} / e_{n-1}^{8}\right|$ and the computational order of convergence $\rho_{n}$. We use the formula by Jay [10] given as:

$$
\rho_{n} \approx \frac{\log \left|f\left(x_{n+1}\right) / f\left(x_{n}\right)\right|}{\log \left|f\left(x_{n}\right) / f\left(x_{n-1}\right)\right|}
$$

in order to calculate $\rho_{n}$.

We have done our calculations with several number of significant digits (minimum 1000 significant digits) to minimize the round off error. We calculate the values of all the constants and functional residuals up to several number of significant digits but due to the limitations. We display the value of errors per iterations and absolute residual errors in the function up to 9 decimal digits with exponent power in Tables $1-5$. In Table 1 , these four typical methods have been successfully applied to the test functions $f_{1}-f_{4}$ below:

$$
\begin{aligned}
& f_{1}(x)=x^{3}-5.22 x^{2}+9.0825 x-5.2675, m=2, \alpha=1.75 \\
& f_{2}(x)=\frac{x}{1-x}-5 \ln \left(\frac{0.4(1-x)}{0.4-0.5 x}\right)+4.45977, m=1, \alpha=0.757396246 \\
& f_{3}(x)=x^{4}+11.50 x^{3}+47.49 x^{2}+83.06325 x+51.23266875, m=2, \alpha=-2.85 \\
& f_{4}(x)=(\cos (x)-x)^{3}, m=3, \alpha=0.739085133 .
\end{aligned}
$$

We see that these examples have different applications in Chemistry. Let us describe the phenomena as follows:

Example 1 Van der Waals Equation of State, whose expression is

$$
\left(P+\frac{a_{1} n^{2}}{V^{2}}\right)\left(V-n a_{2}\right)=n R T,
$$

explains the behavior of a real gas by taking in the ideal gas equations two more parameters, $a_{1}$ and $a_{2}$, specific for each gas. In order to determine the volume $V$ of the gas in terms of the remaining parameters, we are required to solve the nonlinear equation in $\mathrm{V}$.

$$
P V^{3}-\left(n a_{2} P+n R T\right) V^{2}+a_{1} n^{2} V-a_{1} a_{2} n^{3}=0 .
$$

Given the constants $a_{1}$ and $a_{2}$ of a particular gas, one can find values for $n, P$ and $T$, such that this equation has three real roots. By using the particular values, we obtain the following nonlinear function

$$
f_{1}(x)=x^{3}-5.22 x^{2}+9.0825 x-5.2675
$$

having three roots out of which one is a multiple zero $\alpha=1.75$ of multiplicity of order two and other one simple zero $\xi=1.72$. However, our desired zero is $\alpha=1.75$. We considered initial guess $x_{0}=1.8$ for this problem. 
Table 2: Comparison of methods for $f_{1}(x)$

\begin{tabular}{lllll}
\hline \multicolumn{4}{l}{$f_{1}(x), x_{0}=1.8, m=2, \alpha=1.75$} & \\
\hline$\left|x_{n}-\alpha\right|$ & $\left|x_{1}-\alpha\right|$ & $\left|x_{2}-\alpha\right|$ & $\left|x_{3}-\alpha\right|$ & $\rho_{n}$ \\
\hline$G M 1$ & $3.077732689(-3)$ & $1.439104082(-6)$ & $1.091883839(-19)$ & 3.914835182 \\
$G M 2$ & $1.050232397(-3)$ & $4.705856570(-11)$ & $4.992888939(-55)$ & 5.977919826 \\
$O M$ & $5.700719507(-4)$ & $1.356336629(-15)$ & $1.675463909(-108)$ & 7.990284720 \\
$M 1$ & $3.881723198(-4)$ & $5.160856712(-17)$ & $5.930141567(-120)$ & 7.992771335 \\
$M 2$ & $4.097456687(-4)$ & $9.751215264(-17)$ & $1.191072740(-117)$ & 7.992231302 \\
$M 3$ & $4.030985318(-4)$ & $8.578320923(-17)$ & $4.272545051(-118)$ & 7.992383207 \\
$M 4$ & $3.180624956(-4)$ & $6.347458544(-18)$ & $1.812641621(-127)$ & 7.994648047 \\
\hline
\end{tabular}

In Table 2 we show the numerical results obtained by applying the different methods for approximating the multiple solution of $f_{1}(x)=0$. The obtained values confirm the theoretical results.

Regarding the dynamical behavior of function $f_{1}(x)$, it can be observed in Figures 1 and 2 that, for some methods the only basin of attraction is that of the multiple root. The dynamical planes that appear in this section have been generated by using the routines published in [4]. We have used a mest of $400 \times 400$ points in the region of the complex plane $[-100,100] \times[-100,100]$. We paint in orange the points whose orbit converges to the multiple root and in black whose points whose orbit converges to another thing (strange fixed points, cycles, etc.) or diverges. We work with a tolerance of $10^{-3}$ and a maximum number of 80 iterations. The multiple root is represented in the different figures by a white star.

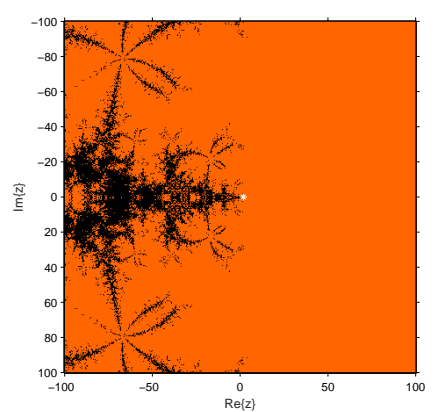

(a) GM1

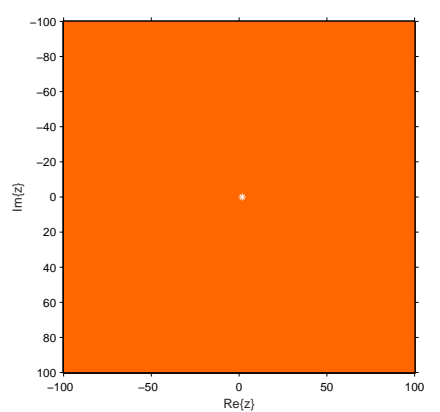

(b) GM2

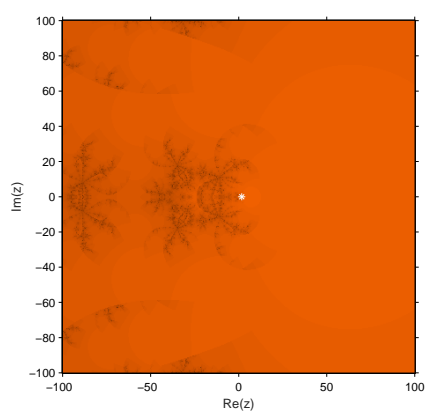

(c) $O M$

Fig. 1: Basin of attractions for function $f_{1}(x)$

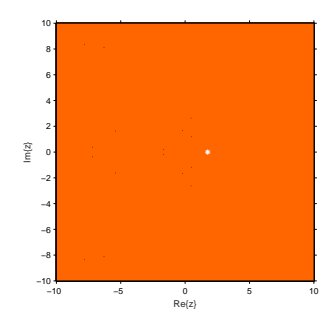

(a) $M 1$

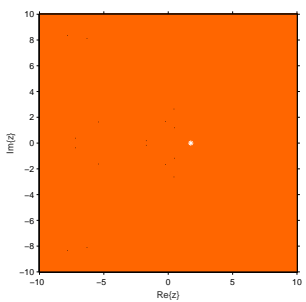

(b) $M 2$

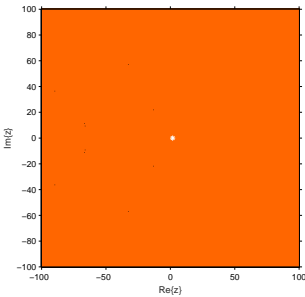

(c) $M 3$

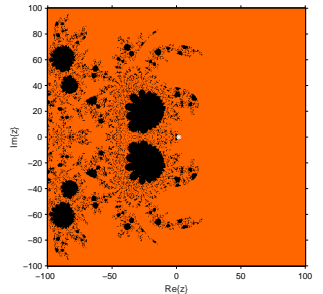

(d) $M 4$

Fig. 2: Basin of attractions for function $f_{1}(x)$ 
Table 3: Comparison of methods for $f_{2}(x)$

\begin{tabular}{lllll}
\hline \multicolumn{4}{l}{$f_{2}(x), x_{0}=0.76, m=1, \alpha=0.757396246$} & \\
\hline$\left|x_{n}-\alpha\right|$ & $\left|x_{1}-\alpha\right|$ & $\left|x_{2}-\alpha\right|$ & $\left|x_{3}-\alpha\right|$ & $\rho_{n}$ \\
\hline$G M 1$ & $6.746046923(-7)$ & $3.258788383(-21)$ & $1.774563094(-78)$ & 3.999998137 \\
$G M 2$ & $5.354140101(-9)$ & $4.610283706(-43)$ & $1.879123364(-247)$ & 5.999999990 \\
$O M$ & $6.001645913(-11)$ & $5.072586488(-72)$ & $1.320998094(-560)$ & 7.999999999 \\
$M 1$ & $5.114097140(-11)$ & $1.600842565(-72)$ & $1.475658388(-564)$ & 7.999999999 \\
$M 2$ & $7.672103880(-11)$ & $5.903530034(-71)$ & $7.255881828(-552)$ & 7.999999999 \\
$M 3$ & $7.658677908(-11)$ & $5.821386344(-71)$ & $6.486454366(-552)$ & 7.999999999 \\
$M 4$ & $2.967992578(-11)$ & $1.141632108(-74)$ & $5.470576454(-582)$ & 7.999999999 \\
\hline
\end{tabular}

Example 2 Fractional Conversion in a Chemical Reactor.

Let us consider the following expression (please, see [14] for more details)

$$
f_{2}(x)=\frac{x}{1-x}-5 \log \left[\frac{0.4(1-x)}{0.4-0.5 x}\right]+4.45977 .
$$

In this equation $x$ represents the fractional conversion of species A in a chemical reactor. Since, there will be no physical meaning of above fractional conversion if $x$ is less than zero or greater than one. So, $x$ is bounded in the region $0 \leq x \leq 1$. Our required simple root to this problem is $\alpha=0.75739624625375387945$. Moreover, it is interesting to note that $\mathrm{f}(\mathrm{x})$ is undefined in the region $0.8 \leq x \leq 1$ which is very close to our desired root. Furthermore, there are some other properties to this function which make the solution more difficult. The derivative of the above expression will be very close to zero in the region $0 \leq x \leq 0.5$ and there is an infeasible solution for $x=1.098$. So, the initial approximation is taken as $x_{0}=0.76$.

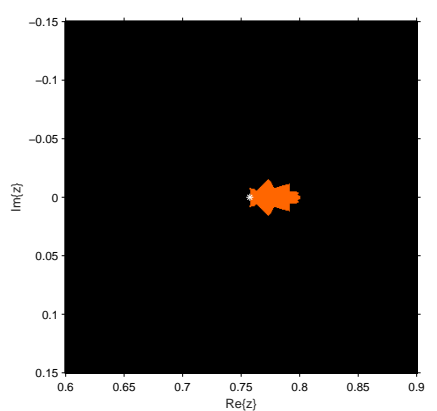

(a) $G M 1$

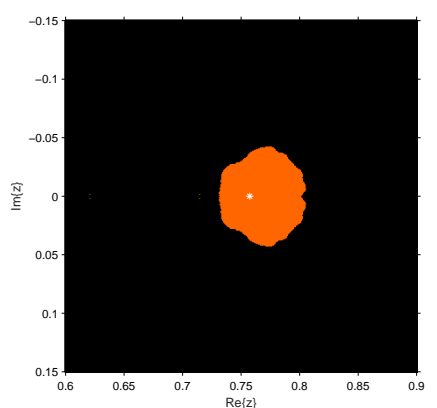

(b) GM2

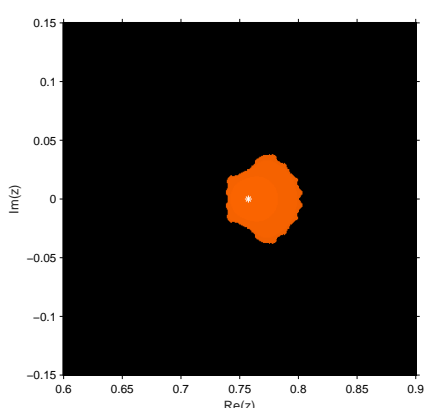

(c) $O M$

Fig. 3: Basin of attractions for function $f_{2}(x)$

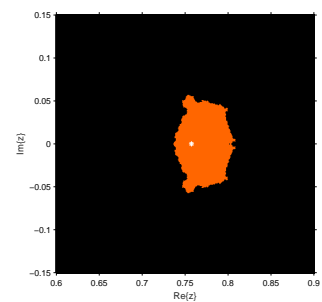

(a) $M 1$

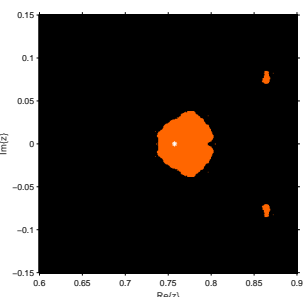

(b) $M 2$

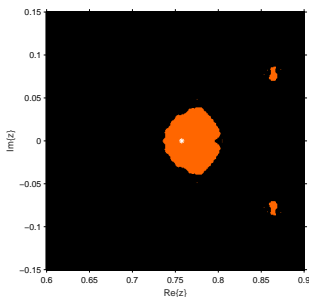

(c) $M 3$

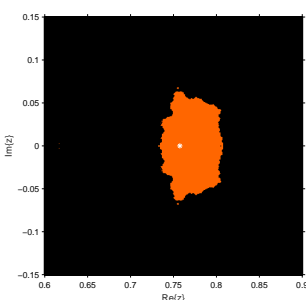

(d) $M 4$

Fig. 4: Basin of attractions for function $f_{2}(x)$ 
Table 4: Comparison of methods for $f_{3}(x)$

\begin{tabular}{lllll}
\hline \multicolumn{4}{l}{$f_{3}(x), x_{0}=0.76, m=1, \alpha=0.757396246$} & \\
\hline$\left|x_{n}-\alpha\right|$ & $\left|x_{1}-\alpha\right|$ & $\left|x_{2}-\alpha\right|$ & $\left|x_{3}-\alpha\right|$ & $\rho_{n}$ \\
\hline$G M 1$ & $2.191617424(-3)$ & $2.388130175(-7)$ & $1.868020089(-29)$ & 5.578713509 \\
$G M 2$ & $4.434881447(-3)$ & $2.040917706(-6)$ & $1.003474537(-37)$ & 9.382151166 \\
$O M$ & $5.957397881(-3)$ & $5.050121091(-6)$ & $2.869820096(-46)$ & 13.10205586 \\
$M 1$ & $9.172403924(-3)$ & $1.902277775(-5)$ & $5.834785506(-42)$ & 13.60857768 \\
$M 2$ & $9.169540439(-3)$ & $1.900995429(-5)$ & $5.803772849(-42)$ & 13.60853336 \\
$M 3$ & $9.172404158(-3)$ & $1.902277880(-5)$ & $5.835168006(-42)$ & 13.60856714 \\
$M 4$ & $1.966472933(-2)$ & $2.173523392(-4)$ & $8.533280914(-34)$ & 15.03163215 \\
\hline
\end{tabular}

Table 3 shows the numerical results obtained for $f_{2}(x)$. We can observe the similarity among all the results of the eighth-order schemes and that COC approaches very good the theoretical order of convergence, except for the scheme GM1. Figures 3 and 4 show the basins of attraction of the different methods on $f_{2}(x)$. In this example, we can observe that the set of good initial approximations is small in all cases.

Example 3 Continuous Stirred Tank Reactor (CSTR)

Consider the isothermal continuous stirred tank reactor (CSTR). Components A \& R are fed to the reactor at rates of $\mathrm{Q}$ and $\mathrm{q}-\mathrm{Q}$ respectively. The following reaction scheme develops in the reactor (see [5]):

$$
\begin{aligned}
& A+R \rightarrow B \\
& B+R \rightarrow C \\
& C+R \rightarrow D \\
& C+R \rightarrow E .
\end{aligned}
$$

The problem was analyzed by Douglas [6] in order to design simple feedback control systems. In the analysis, he gave the following equation for the transfer function of the reactor:

$$
K_{C} \frac{2.98(x+2.25)}{(s+1.45)(s+2.85)^{2}(s+4.35)}=-1 \text {, }
$$

where $K_{C}$ is the gain of the proportional controller. The control system is stable for values of $K_{C}$ that yields roots of the transfer function having negative real part. If we choose $K_{C}=0$ we get the poles of the open-loop transfer function as roots of the nonlinear equation:

$$
f_{3}(x)=x^{4}+11.50 x^{3}+47.49 x^{2}+83.06325 x+51.23266875=0
$$

given as: $x=-1.45,-2.85,-2.85,-4.35$.

So, we see that there is one multiple roots with multiplicity 2 . We take $m=2$ and $x_{0}=-3$.

Table 4 shows the numerical results for this example and in Figures 5 and 6 the corresponding basis of attraction are painted.

Example 4 We assume another standard test problem involving trigonometric function as:

$$
f_{4}(x)=(\cos (x)-x)^{3}
$$

The above function has a multiple zero at $\alpha=0.73908513321516064165$ of multiplicity 3 with initial guess 1.0 .

Finally, Table 5 shows the numerical results for the equation $f_{4}(x)=0$ and in Figures 7 and 8 the corresponding basis of attraction are painted. 


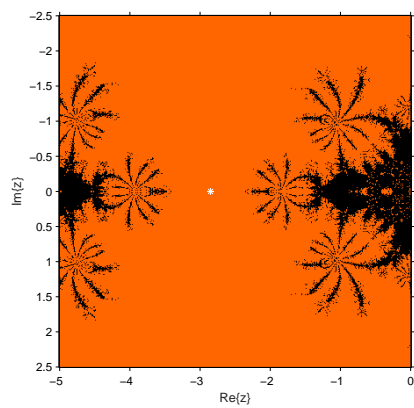

(a) GM1

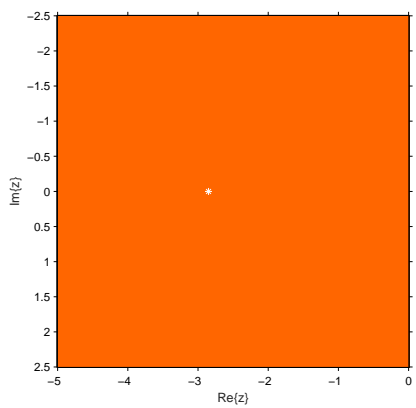

(b) GM2

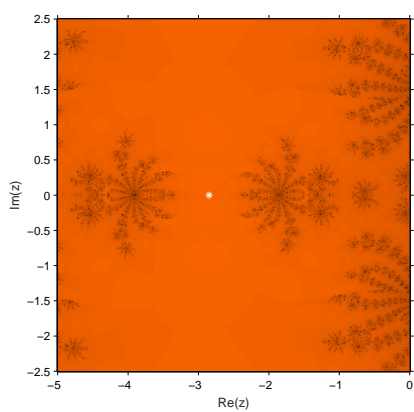

(c) $O M$

Fig. 5: Basin of attractions for function $f_{3}(x)$

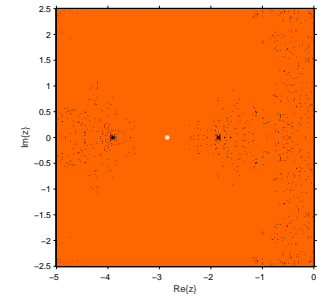

(a) $M 1$

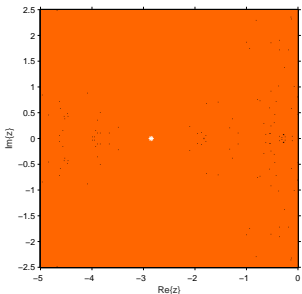

(b) $M 2$

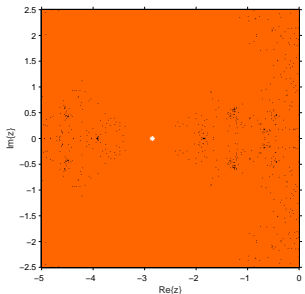

(c) $M 3$

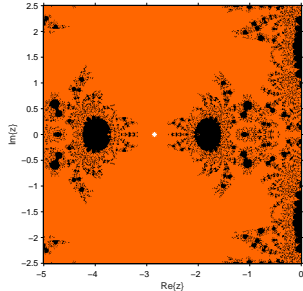

(d) $M 4$

Fig. 6: Basin of attractions for function $f_{3}(x)$

Table 5: Comparison of methods for $f_{4}(x)$

\begin{tabular}{|c|c|c|c|c|}
\hline \multicolumn{5}{|c|}{$f_{4}(x), x_{0}=0.76, m=1, \alpha=0.757396246$} \\
\hline$\left|x_{n}-\alpha\right|$ & $\left|x_{1}-\alpha\right|$ & $\left|x_{2}-\alpha\right|$ & $\left|x_{3}-\alpha\right|$ & $\rho_{n}$ \\
\hline$G M 1$ & $1.566592682(-4)$ & $4.132424869(-17)$ & $2.001658027(-67)$ & 3.999980164 \\
\hline$G M 2$ & $2.55308875(-6)$ & $6.835881397(-36)$ & $2.518668789(-213)$ & 5.999999784 \\
\hline$O M$ & $8.481354394(-8)$ & $4.488396982(-59)$ & $2.761212765(-469)$ & 7.999999994 \\
\hline$M 1$ & $4.905393922(-8)$ & $4.062521585(-61)$ & $8.990216944(-486)$ & 7.999999996 \\
\hline$M 2$ & $5.525400401(-8)$ & $1.249500760(-60)$ & $8.545133533(-482)$ & 7.999999995 \\
\hline$M 3$ & $5.512544243(-8)$ & $1.226431201(-60)$ & $7.361599398(-482)$ & 7.999999996 \\
\hline$M 4$ & $2.997912648(-8)$ & $4.556082715(-63)$ & $1.296500510(-501)$ & 7.999999998 \\
\hline
\end{tabular}

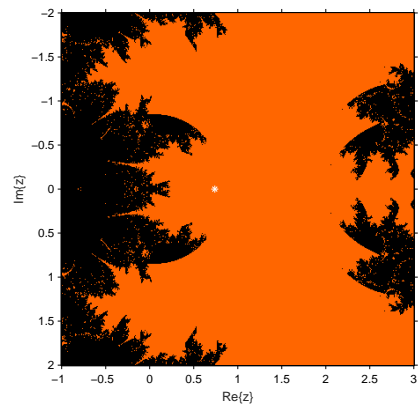

(a) $G M 1$

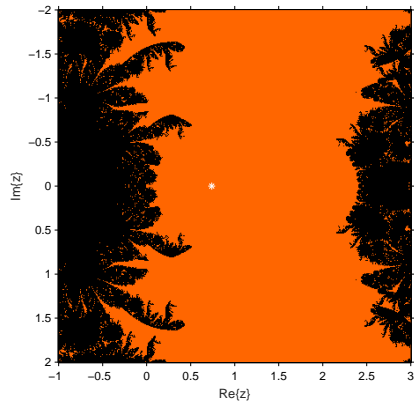

(b) $G M 2$

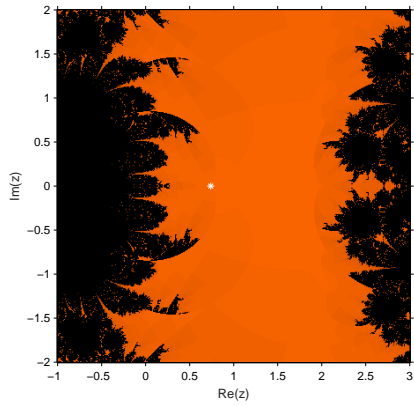

(c) $O M$

Fig. 7: Basin of attractions for function $f_{4}(x)$ 


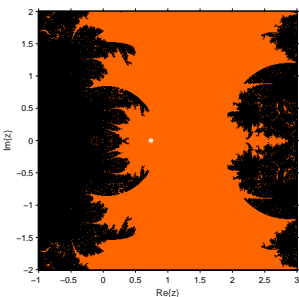

(a) $M 1$

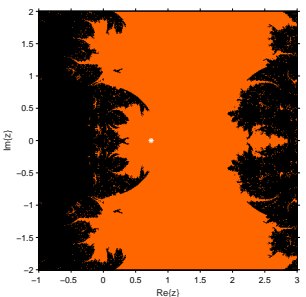

(b) $M 2$

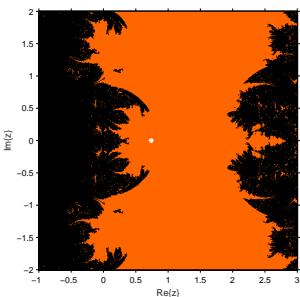

(c) $M 3$

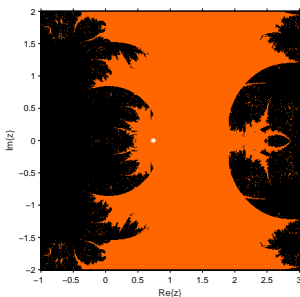

(d) $M 4$

Fig. 8: Basin of attractions for function $f_{4}(x)$

\section{Conclusion}

In this paper, we have proposed a family of iterative methods for solving nonlinear equations for multiple roots with known multiplicity. The family of methods include two free parameters and three weight functions involving function-to-function ratio. The methods involve only one derivative evaluation. The selection of the parameters and weight functions yields optimal eighth order convergent methods for multiple roots. In addition, the numerical results of some chemical problems show that the proposed methods namely M1-M4 have better performance as compared with other similar methods. The dynamical planes of the operators that describe the methods on these problems give us information about the set of initial approximations with guarantee of convergence

\section{References}

1. R. Behl, A. Cordero, S.S. Motsa, J.R. Torregrosa, On developing fourth-order optimal families of methods for multiple roots and their dynamics, Appl. Math. Comput. 265(15) 520-532 (2015)

2. R. Behl, A. Cordero, S.S. Motsa, J.R. Torregrosa, V. Kanwar, An optimal fourth-order family of methods for multiple roots and its dynamics, Numer. Algor. 71(4) 775-796 (2016)

3. R. Behl, Alicia Cordero, Sandile S. Motsa and Juan R. Torregrosa, An eighth-order family of optimal multiple root finders and its dynamics, Numer. Algor. In Press, (2017)

4. F.I. Chicharro, A. Cordero, J. R. Torregrosa, Drawing dynamical and parameters planes of iterative families and methods, Sci. World J. 2013, ID 780153, 11 pages 2013

5. A. Constantinides, N. Mostoufi, Numerical Methods for Chemical Engineers with MATLAB Applications, Prentice Hall PTR, New Jersey, 1999

6. J. M. Douglas, Process Dynamics and Control, Vol. 2, Prentice Hall, Englewood Cliffs, New Jersey, 1972

7. Y.H. Geum, Y.I. Kim, B. Neta, A class of two-point sixth-order multiple-zero finders of modified double-Newton type and their dynamics, Appl. Math. Comput. 270 387-400 (2015)

8. Y.H. Geum, Y.I. Kim, B. Neta, A sixth-order family of three-point modified Newton-like multiple-root finders and the dynamics behind their extraneous fixed points, Appl. Math. Comput. 283 120-140 (2016)

9. J.L. Hueso, E. Martinez, C. Teruel, Determination of multiple roots of nonlinear equations and applications, J. Math. Chem. 53 880-892 (2015)

10. L.O. Jay, A note on Q-order of convergence. BIT Numer. Math. $41422-429$ (2001)

11. S. Li, X. Liao, L. Cheng, A new fourth-order iterative method for finding multiple roots of nonlinear equations, Appl. Math. Comput. 215 1288-1292 (2009)

12. S.G. Li, L.Z. Cheng, B. Neta, Some fourth-order nonlinear solvers with closed formulae for multiple roots, Comput. Math. Appl. 59 126-135 (2010)

13. B. Liu, X. Zhou, A new family of fourth-order methods for multiple roots of nonlinear equations, Non. Anal. Model. Cont. 18(2) 143-152 (2013)

14. M. Shacham, Numerical solution of constrained nonlinear algebraic equations, Int. J. Numer. Method Eng. $231455-1481$ (1986)

15. M. Sharifi, D.K.R. Babajee, F. Soleymani, Finding the solution of nonlinear equations by a class of optimal methods, Comput. Math. Appl. 63 764-774 (2012)

16. J.R. Sharma, R. Sharma, Modified Jarratt method for computing multiple roots, Appl. Math. Com-put. $217878-881$ (2010)

17. F. Soleymani, D.K.R. Babajee, T. Lofti, On a numerical technique forfinding multiple zeros and its dynamic, J. Egypt. Math. Soc. $21346-353(2013)$

18. F. Soleymani, D.K.R. Babajee, Computing multiple zeros using a class of quartically convergent methods, Alex. Eng. J. 52 $531-541(2013)$

19. R. Thukral, A new family of fourth-order iterative methods for solving nonlinear equations with multiple roots, J. Numer. Math. Stoch. 6(1) 37-44 (2014)

20. R. Thukral, Introduction to higher-order iterative methods for finding multiple roots of nonlinear equations, J. Math. 2013 (2013) Article ID 404635, 3 pages.

21. X. Zhou, X. Chen, Y. Song, Constructing higher-order methods for obtaining the muliplte roots of nonlinear equations, J. Comput. Math. Appl. 235 4199-4206 (2011) 
22. X. Zhou, X. Chen, Y. Song, Families of third and fourth order methods for multiple roots of nonlinear equations, Appl. Math. Comput. 219 6030-6038 (2013) 